\title{
The Minimum Cost Path Finding Algorithm Using a Hopfield Type Neural Network
}

\author{
Sun-Gi Hong, Sung-Woo Kim, and Ju-Jang Lee \\ Department of Electrical Engineering \\ Korea Advanced Institute of Science and Technology \\ 373-1 Kusong-dong, Yusong-gu, Taejon 305-701, Korea \\ FAX: +82-42-869-3410 E-mail: jjlee@ee.kaist.ac.kr
}

\begin{abstract}
Recently neural networks have been proposed as new computational tools for solving constrained optimization problems. In this paper the minimum cost path finding algorithm is proposed by using a Hopfield type neural network. In order to design a Hopfield type neural network, an energy function must be defined at first. To achieve this, the concept of a vector-represented network is used to describe the connected path. Through simulations, it will be shown that the proposed algorithm works very well in many cases. The local minima problem of a Hopfield type neural network is discussed.
\end{abstract}

\section{INTRODUCTION}

Generally, to find the minimum cost path on the network model is involved in many problems. For this reason the minimum cost path finding problem has been the subject of intensive research for many years. Hence, many algorithms have been proposed, for example, Dijkstra's algorithm and so on [1]. However, these algorithms have the shortcomings that the computational burden is getting larger as the number of nodes increases on the network model. Recently neural networks have been proposed as new computational tools for solving constrained optimization problems. Since the minimum cost path finding problem can be regarded as a constrained optimization problem, we propose the minimum cost path finding algorithm using a Hopfield type neural network in this paper. To design a Hopfield type neural network, an appropriate energy function must be defined at first so that the minimum cost path can be decoded from the final state of the neural network. To achieve this, we assume that the network model is given in the form of vectors between the nodes without loss of generality. Using this vector-represented network model, the constraints for the minimum cost path is presented in this paper. After finding the dynamic equation of each neuron based on the steepest descent method, the electrical circuit can be implemented by using the obtained parameters as shown in Fig. 1. In this paper, through the 
numerical analysis, it will be shown that the proposed algorithm can solve the minimum cost path problems.

Generally, a Hopfield type neural network has the local minima problem because the dynamics of each neuron is obtained, based on the steepest descent method. In order to overcome this shortcomings, a surface of the energy function must be designed gracefully by proper tuning the weighted coefficients of the energy function. This tuning scheme has been proposed by Mustafa $\mathrm{K}$. Mehmet Ali and Faouzi Kamoun and is discussed in this paper [2].

This paper is organized as follows:

The next section gives a brief review of the application of a Hopfield type neural network to solve discrete combinatorial optimization problems. In section III, a neural network architecture based on the Hopfield model is proposed for solving the minimum cost path finding problem. The main steps involved in the design of the proposed model are described. In section IV, it will be shown that the proposed model works well in many cases, through computer simulations. Finally we draw some conclusions in section $\mathrm{V}$.

\section{HOPFIELD TYPE NEURAL NETWORK}

The use of neural networks to solve constrained optimization problems was initiated by Hopfield and Tank [3]. A Hopfield type neural network has been applied to solve discrete combinatorial optimization problems, (for example, the Traveling Salesman Problem (TSP)) because of its computational power. A Hopfield type neural network can be implemented by the electrical circuit as shown in Fig. 1. This circuit is based on the model of a biological neural network. Each neuron is modeled as a nonlinear device (operational amplifier) with a saturated monotonic increasing function relating the output $V_{i}$ of the $i$ th neuron to its input $U_{i}$. The example of a typical characteristic function of the nonlinear device is as follows:

$$
V_{i}=g_{i}\left(U_{i}\right)=\frac{1}{1+e^{-\lambda_{i} U_{i}}}
$$

Using the above equation, the output $V_{i}$ is allowed to take on any value between 0 and 1 . The synaptic connections between neurons are represented as resistive components which can be fully described through the matrix $T=\left[T_{i j}\right]$, also known as the connection matrix of the network. As shown in Fig. 1, each neuron is biased by an external current $I_{i}$, which could represent actual data provided by user to the neural network. The dynamics of a Hopfield type neural network is described as follows:

$$
\frac{d U_{i}}{d t}=-\frac{U_{i}}{\tau_{i}}-\sum_{j=1}^{N} T_{i j} V_{j}-I_{i}
$$

where $\tau_{i}$ is the circuit's time constant.

Hopfield [3] has shown that if the connection matrix $T$ is symmetric and the gains of the nonlinear devices are sufficiently high (i.e. $\lambda_{i} \rightarrow \infty$ ) then the dynamics of the neurons moves to the stable state in the gradient descent direction of the quadratic energy function (3).

$$
E=\frac{1}{2} \sum_{i=1}^{N} \sum_{j=1}^{N} T_{i j} V_{i} V_{j}+\sum_{i=1}^{N} I_{i} V_{i}
$$

Hopfield [3] has also shown that if the state of the space over which the circuit operates is the interior of the $N$-dimensional hypercube 
defined by $V_{i}=0$ or 1 , i.e. when $\lambda_{i}=\infty$, and the diagonal elements $T_{i i}$ are 0 , then the stable states of the network correspond to those locations in the discrete space consisting of the $2^{N}$ comers of this hypercube which minimize $E$ (3). In terms of the generalized energy function , the dynamics of the $i$ th neuron is described as follows:

$$
\frac{d U_{i}}{d t}=-\frac{U_{i}}{\tau_{i}}-\frac{\partial E}{\partial V_{i}}
$$

Therefore, to apply a Hopfield type neural network to the minimum cost finding problem the energy function is required.

\section{THE MINIMUM COST PATH} FINDING ALGORITHM

The vector-represented network model can be defined as a directed graph $G=(\bar{N}, \bar{A})$, with $N$ nodes and $l$ arcs. Corresponding to each arc $(i, j)$ there is a nonnegative number $C_{i j}$ which represents the cost value (length, transit time, etc.) from node $i$ to node $j$. We assign the number to each node from 0 to $N-1$. Especially a source and a destination are assigned $O$ and $N-1$ respectively. Let a directed path $P^{O N-1}$ be an ordered sequence of nodes connecting $O$ to $N-I$ [2].

$$
P^{0 N-1}=(0, i, j, \cdots, k, N-1)
$$

Then the total cost of this path will be equal to $C_{0 i}+C_{i j}+\cdots+C_{k N-1}$. Hence, the minimum cost path can be found among all possible directed paths.

To formulate the minimum cost path finding problem in terms of a Hopfield type neural network, an appropriate energy function must be defined at first. To achieve this, the proposed Hopfield type neural network is organized in $N(N-I) / 2$ neurons. Each neuron is described by double indices $(i, j)$, where the subscript $i$ and $j$ denote node numbers. A neuron which represents $(i, j)$ is characterized by its output $V_{i j}$ and is defined as follows:

$$
V_{i j}= \begin{cases}1 & \begin{array}{l}
\text { if the arc from node } i \text { to node } \\
j \text { is in the minimum cost path (6) }
\end{array} \\
0 & \text { otherwise }\end{cases}
$$

To eliminate all nonexistent arcs from the solution the following parameter is introduced.

$$
\rho_{i j}= \begin{cases}1 & \text { if the arc from node } i \text { to node } \\ j \text { does not exist } & \text { otherwise }\end{cases}
$$

To express the connecting condition for the minimum cost path, which means the feasible path must be perfectly connected through the nodes, the following assumption is required.

\section{Assumption 1}

A directed graph $G=(\bar{N}, \bar{A})$ is represented as the form of vectors between nodes.: the network model is given in the form of the vectorrepresented network.

Since each node of a directed graph has the coordinate for $X-Y$ plane, the vector between the nodes can be easily obtained. Let $\left(x_{i}, y_{i}\right)$ be the coordinate of node $i$, then the vector between node $i$ and node $j$ is defined as follows:

$$
\overrightarrow{d_{i j}}= \begin{cases}\left(x_{j}, y_{j}\right)-\left(x_{i}, y_{i}\right) & \text { if the arc from node } \\
\overrightarrow{0} & \begin{array}{l}
i \text { to node } j \text { exists } \\
\text { otherwise }
\end{array}\end{cases}
$$

To describe the vector-represented network, the 
directional factor $\alpha_{i j}$ is also defined as follows:

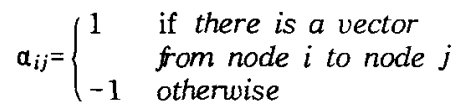

Using the vector $a_{i j} \overrightarrow{d_{i j}}$, a vector-represented network can be described easily as shown in Fig. 2. The connecting condition is represented as the following equation using the parameters defined in equation (8), (9).

$$
\sum_{i=0}^{N-1} \sum_{j=i+1}^{N-1} a_{i j} \overrightarrow{d_{i j}} V_{i j}=\overrightarrow{d_{0 N-1}}
$$

Equation (10) is not "if and only if" condition for representing the connected path from a source to a destination because the vector $\vec{d}_{i j}$ is not unique. To obtain "if and only if" condition, the following function is introduced.

$$
F(i)=\sum_{k=0}^{i-1} V_{k i}+\sum_{k=i+1}^{N-1} V_{i k}
$$

The value of the function $F(i)$ means the total sum of the connected paths at node $i$ when the dynamics of neurons reaches the stable state. If the following equation is satisfied, then the connected path between a source and a destination is generated.

$$
\begin{cases}F(i) F(j) V_{i j}=2 & \text { if node } i \text { is a } \\ & \text { source or node } j \\ & \text { is a destination }\end{cases}
$$

where $(i, j) \in[0, N-1] / i<j$.

The example of equation (12) is depicted in Fig. 3. Since a source node and a destination node must have the only one connected path respectively, the following condition is also needed in addition to equation (12).

$$
\sum_{j=1}^{N-1} V_{0 j}=1 \quad \text { at a source node }
$$

$$
\sum_{i=0}^{N-2} V_{i N-1}=1 \text { at a destination }
$$

Therefore, according to equation (10), (12), (13), (14) the energy function can be formulated as follows:

(i) If node $i$ is the starting point or node $j$ is the destination

$$
\begin{aligned}
E= & \mu_{1} \sum_{i=0}^{N-1} \sum_{j=i+1}^{N-1} C_{i j} V_{i j}+\mu_{2} \sum_{i=0}^{N-1} \sum_{j=i+1}^{N-1} \rho_{i j} V_{i j} \\
& +\mu_{3}\left\|\sum_{i=0}^{N-1} \sum_{j=i+1}^{N-1} \alpha_{i j} \overrightarrow{d_{i j}} V_{i j}-\overrightarrow{d_{0 N-1}}\right\|^{2} \\
& +\mu_{4}\left(\sum_{i=0}^{N-1} \sum_{j=i+1}^{N-1} F(i) F(j) V_{i j}-2\right)^{2} \\
& +\mu_{5}\left(\sum_{j=1}^{N-1} V_{0 j}-1\right)^{2}+\mu_{6}\left(\sum_{i=0}^{N-2} V_{i N-1}-1\right)^{2} \\
& +\mu_{7} \sum_{i=0}^{N-1} \sum_{j=i+1}^{N-1} V_{i j}\left(1-V_{i j}\right)
\end{aligned}
$$

(ii) otherwise

$$
\begin{aligned}
E= & \mu_{1} \sum_{i=0}^{N-1} \sum_{j=i+1}^{N-1} C_{i j} V_{i j}+\mu_{2} \sum_{i=0}^{N-1} \sum_{j=i+1}^{N-1} \rho_{i j} V_{i j} \\
& +\mu_{3}\left\|\sum_{i=0}^{N-1} \sum_{j=i+1}^{N-1} a_{i j} \overrightarrow{d_{i}} V_{i j}-\overrightarrow{d_{0 N-1}}\right\|^{2} \\
& +\mu_{4}\left(\sum_{i=0}^{N-1} \sum_{j=i+1}^{N-1} F(i) F(j) V_{i j}-4\right)^{2} \\
& +\mu_{5}\left(\sum_{j=1}^{N-1} V_{0 j}-1\right)^{2}+\mu_{6}\left(\sum_{i=0}^{N-2} V_{i N-1}-1\right)^{2} \\
& +\mu_{7} \sum_{i=0}^{N-1} \sum_{j=i+1}^{N-1} V_{i j}\left(1-V_{i j}\right)
\end{aligned}
$$

where $\|\cdot\|$ is the Euclidean norm.

In equation (15) and (16) the $\mu_{1}$ term minimizes the total cost of a path by taking into account the cost of existing arcs. The $\mu_{2}$ term prevents nonexistent arcs from being included in the chosen path. The $\mu_{3}, \mu_{4}, \mu_{5}$, and $\mu_{6}$ terms are zero if the minimum cost path is connected from a source node and a destination through nodes The $\mu_{7}$ term pushes the state of a Hopfield type neural network to converge to one of the $2^{N(N-1) / 2}$ comers of the hypercube, defined by $V_{i j} \in[0,1]$ [2] 
Using equation (1) and (4) the dynamics of a Hopfield type neural network can be obtained as follows:

$$
\begin{aligned}
& V_{i j}=g_{i j}\left(U_{i j}\right)=\frac{1}{1+e^{-\lambda_{i j} U_{i j}}} \\
& \forall(i, j) \in[0, N-1] / i<j
\end{aligned}
$$

(i) If node $i$ is a source or node $j$ is a destination

$$
\begin{aligned}
\frac{d U_{i j}}{d t}= & \frac{-U_{i j}}{\tau}-\mu_{1} C_{i j}-\mu_{2} \rho_{i j}-2 \mu_{3}\left\{\left(\sum_{i=0}^{N-1}\right.\right. \\
& \left.\sum_{j=i+1}^{N-1} \alpha_{i j} d_{x i j} V_{i j}-d_{x 0 N-1}\right) \alpha_{i j} d_{x i j}+( \\
& \left.\left.\sum_{i=0}^{N-1} \sum_{j=i+1}^{N-1} \alpha_{i j} d_{y i j} V_{i j}-d_{y 0 N-1}\right) \alpha_{i j} d_{y i j}\right\} \\
& -2 \mu_{4}\left(\sum_{i=0}^{N-1} \sum_{j=i+1}^{N-1} F(i) F(j) V_{i j}-2\right)\{ \\
& \left.F(i) F(j)+F(j) V_{i j}+F(i) V_{i j}\right\}-2 \\
& \mu_{5}\left(\sum_{j=1}^{N-1} V_{0 j}-1\right) \delta_{0 i}-2 \mu_{6}\left(\sum_{i=0}^{N-2} V_{i N-\ell}\right. \\
& -1) \delta_{j N-1}-\mu_{7}\left(1-2 V_{i j}\right) \\
& (i, j) \in[0, \quad N-1] / i<j
\end{aligned}
$$

(ii) otherwise

$$
\begin{aligned}
\frac{d U_{i j}}{d t}= & \frac{-U_{i j}}{\tau}-\mu_{1} C_{i j}-\mu_{2} \rho_{i j}-2 \mu_{3}\left\{\left(\sum_{i=0}^{N-1}\right.\right. \\
& \left.\sum_{j=i+1}^{N-1} \alpha_{i j} d_{x i j} V_{i j}-d_{x 0 N-1}\right) \alpha_{i j} d_{x i j}+( \\
& \left.\left.\sum_{i=0}^{N-1} \sum_{j=i+1}^{N-1} \alpha_{i j} d_{y i j} V_{i j}-d_{y 0 N-1}\right) \alpha_{i j} d_{y i j}\right\} \\
& -2 \mu_{4}\left(\sum_{i=0}^{N-1} \sum_{j=i+1}^{N-1} F(i) F(j) V_{i j}-4\right)\{ \\
& \left.F(i) F(j)+F(j) V_{i j}+F(i) V_{i j}\right\}-2 \\
& \mu_{5}\left(\sum_{j=1}^{N-1} V_{0 j}-1\right) \delta_{0 i}-2 \mu_{6}\left(\sum_{i=0}^{N-2} V_{i N-1}\right. \\
& -1) \delta_{j N-1}-\mu_{7}\left(1-2 V_{i j}\right) \\
& (i, j) \in[0, N-1] / i<j
\end{aligned}
$$

where $\overrightarrow{d_{i j}}$ is equal to $\left(d_{x i j}, d_{y i j}\right)$ and the function $\delta_{i j}$ is defined as follows:

$$
\delta_{i j}= \begin{cases}1 & \text { if } i=j \\ 0 & \text { otherwise }\end{cases}
$$

Using equation (18) and (19) the connection matrix and the bias current can be obtained. The minimum cost path corresponds to the minimal state of the energy function which is defined in equation (15) and (16). Generally, a Hopfield type neural network has the local minima problem because the dynamics of neurons is based on the gradient descent method. To solve this local minima problem the energy surface is designed gracefully by proper tuning of the $\mu_{i}$ 's weighting coefficients. In order to ensure that the energy function has only one low point and hence provides a graceful descent along the energy surface, the following equation is required [2].

$$
\frac{\partial^{2} E}{\partial V_{i j}^{2}}>0 \quad \forall(i, j)
$$

Using equation (21) the $\mu_{\imath}$ 's weighting coefficients can be obtained. The simulation results for the proposed algorithm will be presented in the next section.

\section{SIMULATION}

To show the effectiveness of the proposed algorithm, the vector-tepresented network shown in Fig. 4 is used. Let the cost value $C_{i j}$ be the distance between node $i$ and node $j$, i.e. $\left|\alpha_{i j} \overrightarrow{d_{i j}}\right|$ where the coordinates of nodes are given in Table II. Then we can easily find the fact that a directed path $P^{0 t}=(0,2,4)$ is the minimum cost path. To obtain the parameters for a Hopfield type neural network, the coefficients of the energy function is chosen as shown in Table I. In equation (17), in order to allow the dynamics of neurons to wander freely in their state space, the value of $\lambda_{i j}$ is chosen as 1 and for simplicity it is assumed that $g_{i j}=g$, all independent of the subscript $(i, j)$. Originally, a 
Hopfield type neural network is implemented by the electrical circuit in the form of Fig. 1. However, as a matter of convenience the time evolution of the state of neurons can be simulated by numerically solving equation (18) and (19). This corresponds to solving a system of $N(N-I) / 2$ nonlinear differential equations, where the variables are the neuron's output voltages $V_{i j}$ 's. Accordingly, the simulation consists of observing and updating the neuron's output voltages at incremental time steps $\delta t$, where $\delta t$ is $0.01 \mathrm{~ms}$ in this paper. In addition, the time constant $\tau$ of each neuron is set to 1 without any loss of generality in equation (18) and (19). The initial value of $U_{i j}$ is assigned between 0 and 1 randomly. The simulation result for Fig. 4 is illustrated in Table III. In Table III, the values of $0,1, \cdots, 4$ in rows mean the number of node $i$ and the values in columns mean the number of node $j$ similarly.

As compared with Fig. 4, the vectorrepresented network shown in Fig. 5 has a different configuration; a directed path $P^{04}=(0,1$, 4) is the minimum cost path, where the coordinates of nodes are identical with the case in Fig. 4 and the cost value $C_{i j}$ is the distance between node $i$ and node $j$. The simulation result for Fig. 5 is shown in Table IV, using the above parameters.

These results show that the proposed algorithm operates well. When the number of nodes becomes larger, the minimum cost path can be obtained through the proper tuning of the weight coefficient $\mu_{i}$.

\section{CONCLUSION}

The minimum cost path finding algorithm on the vector-represented network was proposed, using a Hopfield type neural network. To design a Hopfield type neural network, the energy function, which represents the condition for the minimum cost path, was defined. Using this energy function, the parameters for a Hopfield type neural network could be obtained. Through the simulation, the effectiveness of the proposed algorithm was presented. The local minima problem for a Hopfield type neural network was discussed. It was shown that the proposed algorithın could be applied to many cases by proper tuning the coefficients of the energy function.

\section{REFERENCES}

[1] Robert Sedgewick, "Algorithms," Addison Wesley, 1984.

[2] Mustafa K. Mehmet Ali and Faouzi Kamoun, "Neural Networks for Shortest Path Computation and Routing in Computer Networks," IEEE Trans. on Neural Networks, Vol. 4, No. 6, Nov., 1993.

[3] David W. Tank and John J. Hopfield, "Simple "Neural" Optimization Networks : An A/D Converter, Signal Decision Circuit, and a Linear Programming Circuit," IEEE Trans. on Circuits and Systems, Vol. CAS-33, No. 5 , May 1986. 
Table. I. Coefficients of energy function

\begin{tabular}{|c|c|c|c|c|c|c|}
\hline$\mu_{1}$ & $\mu_{2}$ & $\mu_{3}$ & $\mu_{4}$ & $\mu_{5}$ & $\mu_{6}$ & $\mu_{7}$ \\
\hline 800 & 2000 & 5000 & 4000 & 4000 & 4000 & 500 \\
\hline
\end{tabular}

Table. II. Coordinates of nodes

\begin{tabular}{|c|c|c|c|c|}
\hline 0 & 1 & 2 & 3 & 4 \\
\hline$(1,6)$ & $(4,10)$ & $(6,4)$ & $(10,10)$ & $(13,7)$ \\
\hline
\end{tabular}

Table. III. Result for Fig. 4

\begin{tabular}{|c|c|c|c|c|c|}
\hline & 0 & 1 & 2 & 3 & 4 \\
\hline 0 & $\mathrm{X}$ & 0 & 0 & 0.5 & 0.4 \\
\hline 1 & $\mathrm{X}$ & $\mathrm{X}$ & 0 & 0 & 0 \\
\hline 2 & $\mathrm{X}$ & $\mathrm{X}$ & $\mathrm{X}$ & 0 & 0 \\
\hline 3 & $\mathrm{X}$ & $\mathrm{X}$ & $\mathrm{X}$ & $\mathrm{X}$ & 0 \\
\hline 4 & $\mathrm{X}$ & $\mathrm{X}$ & $\mathrm{X}$ & $\mathrm{X}$ & $\mathrm{X}$ \\
\hline
\end{tabular}

(Initial Condition)

\begin{tabular}{|c|c|c|c|c|c|}
\hline & 0 & 1 & 2 & 3 & 4 \\
\hline 0 & $\mathrm{X}$ & 0 & 1 & 0 & 0 \\
\hline 1 & $\mathrm{X}$ & $\mathrm{X}$ & 0 & 0 & 0 \\
\hline 2 & $\mathrm{X}$ & $\mathrm{X}$ & $\mathrm{X}$ & 0 & 1 \\
\hline 3 & $\mathrm{X}$ & $\mathrm{X}$ & $\mathrm{X}$ & $\mathrm{X}$ & 0 \\
\hline 4 & $\mathrm{X}$ & $\mathrm{X}$ & $\mathrm{X}$ & $\mathrm{X}$ & $\mathrm{X}$ \\
\hline
\end{tabular}

(Iterations $=1000$ )

\begin{tabular}{|c|c|c|c|c|c|}
\hline & 0 & 1 & 2 & 3 & 4 \\
\hline 0 & $\mathrm{X}$ & 0 & 1 & 0 & 0 \\
\hline 1 & $\mathrm{X}$ & $\mathrm{X}$ & 0 & 0 & 0 \\
\hline 2 & $\mathrm{X}$ & $\mathrm{X}$ & $\mathrm{X}$ & 0 & 1 \\
\hline 3 & $\mathrm{X}$ & $\mathrm{X}$ & $\mathrm{X}$ & $\mathrm{X}$ & 0 \\
\hline 4 & $\mathrm{X}$ & $\mathrm{X}$ & $\mathrm{X}$ & $\mathrm{X}$ & $\mathrm{X}$ \\
\hline
\end{tabular}

(Iterations $=2000$ )

\begin{tabular}{|c|c|c|c|c|c|}
\hline & 0 & 1 & 2 & 3 & 4 \\
\hline 0 & $\mathrm{X}$ & 0 & 1 & 0 & 0 \\
\hline 1 & $\mathrm{X}$ & $\mathrm{X}$ & 0 & 0 & 0 \\
\hline 2 & $\mathrm{X}$ & $\mathrm{X}$ & $\mathrm{X}$ & 0 & 1 \\
\hline 3 & $\mathrm{X}$ & $\mathrm{X}$ & $\mathrm{X}$ & $\mathrm{X}$ & 0 \\
\hline 4 & $\mathrm{X}$ & $\mathrm{X}$ & $\mathrm{X}$ & $\mathrm{X}$ & $\mathrm{X}$ \\
\hline
\end{tabular}

$($ Iterations $=3000)$

Table. IV. Result for Fig. 5

\begin{tabular}{|c|c|c|c|c|c|}
\hline & 0 & 1 & 2 & 3 & 4 \\
\hline 0 & $\mathrm{X}$ & 0 & 0.5 & 0.5 & 0.4 \\
\hline 1 & $\mathrm{X}$ & $\mathrm{X}$ & 0.1 & 0 & 0 \\
\hline 2 & $\mathrm{X}$ & $\mathrm{X}$ & $\mathrm{X}$ & 0 & 0 \\
\hline 3 & $\mathrm{X}$ & $\mathrm{X}$ & $\mathrm{X}$ & $\mathrm{X}$ & 1 \\
\hline 4 & $\mathrm{X}$ & $\mathrm{X}$ & $\mathrm{X}$ & $\mathrm{X}$ & $\mathrm{X}$ \\
\hline
\end{tabular}

(Initial Condition)

\begin{tabular}{|c|c|c|c|c|c|}
\hline & 0 & 1 & 2 & 3 & 4 \\
\hline 0 & $\mathrm{X}$ & 0.9 & 0 & 0 & 0.1 \\
\hline 1 & $\mathrm{X}$ & $\mathrm{X}$ & 0 & 0.8 & 1 \\
\hline 2 & $\mathrm{X}$ & $\mathrm{X}$ & $\mathrm{X}$ & 0 & 0.1 \\
\hline 3 & $\mathrm{X}$ & $\mathrm{X}$ & $\mathrm{X}$ & $\mathrm{X}$ & 0 \\
\hline 4 & $\mathrm{X}$ & $\mathrm{X}$ & $\mathrm{X}$ & $\mathrm{X}$ & $\mathrm{X}$ \\
\hline
\end{tabular}

(Iterations $=1000)$

\begin{tabular}{|c|c|c|c|c|c|}
\hline & 0 & 1 & 2 & 3 & 4 \\
\hline 0 & $\mathrm{X}$ & 0.9 & 0 & 0 & 0 \\
\hline 1 & $\mathrm{X}$ & $\mathrm{X}$ & 0 & 0.1 & 1 \\
\hline 2 & $\mathrm{X}$ & $\mathrm{X}$ & $\mathrm{X}$ & 0 & 0 \\
\hline 3 & $\mathrm{X}$ & $\mathrm{X}$ & $\mathrm{X}$ & $\mathrm{X}$ & 0 \\
\hline 4 & $\mathrm{X}$ & $\mathrm{X}$ & $\mathrm{X}$ & $\mathrm{X}$ & $\mathrm{X}$ \\
\hline
\end{tabular}

(Iterations $=2000)$ 


\begin{tabular}{|c|c|c|c|c|c|}
\hline & 0 & 1 & 2 & 3 & 4 \\
\hline 0 & $\mathrm{X}$ & 1 & 0 & 0 & 0 \\
\hline 1 & $\mathrm{X}$ & $\mathrm{X}$ & 0 & 0 & 1 \\
\hline 2 & $\mathrm{X}$ & $\mathrm{X}$ & $\mathrm{X}$ & 0 & 0 \\
\hline 3 & $\mathrm{X}$ & $\mathrm{X}$ & $\mathrm{X}$ & $\mathrm{X}$ & 0 \\
\hline 4 & $\mathrm{X}$ & $\mathrm{X}$ & $\mathrm{X}$ & $\mathrm{X}$ & $\mathrm{X}$ \\
\hline
\end{tabular}

$($ Iterations $=3000)$

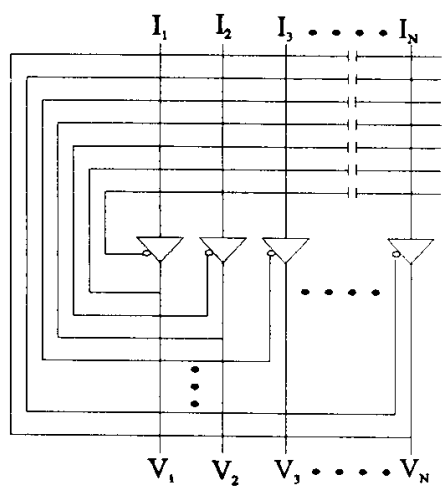

Fig. 1. Hopfield type neural network circuit

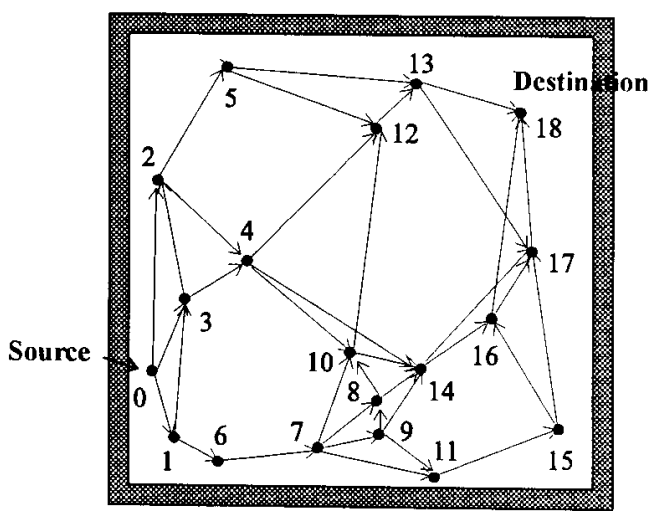

Fig. 2. Vector-represented network

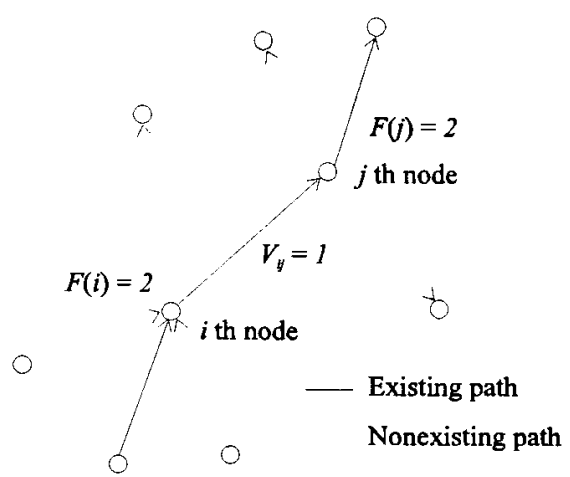

Fig. 3. When $F(i) F(j) V_{i f}=4$

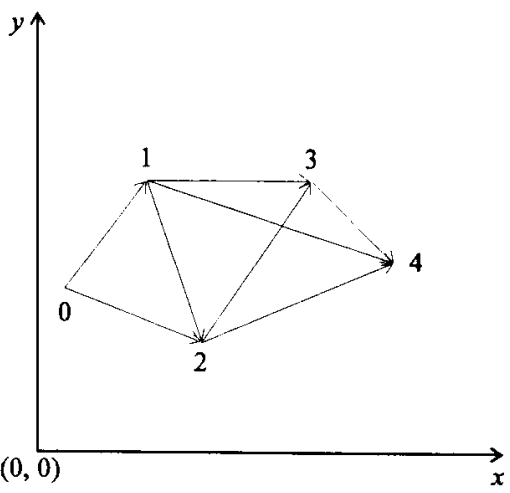

Fig. 4. Vector-tepresented network (0-2-4)

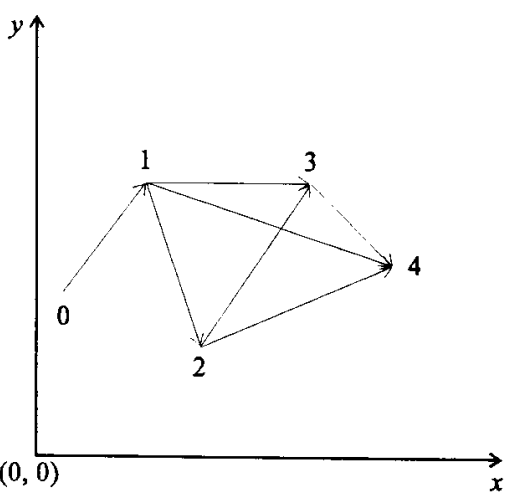

Fig. 5. Vector-represented network (0-1-4) 